DOI 10.19195/2084-2546.24.7

\author{
ANNA KUŹNIK \\ Uniwersytet Wrocławski
}

CARME BESTUÉ

Universitat Autònoma de Barcelona

\title{
Perfiles de prácticas y solución de conflictos emergentes: estudio de un caso de prácticas de traducción jurídica
}

Palabras clave: traducción jurídica — posgrado de traducción jurídica — prácticas perfiles de prácticas - solución de conflictos - coordinación.

\section{Introducción}

El Postgrau de Traducció Jurídica (PTJ) es una formación en la que participan profesores con experiencia profesional contrastada y perfiles muy variados (abogados y traductores en ejercicio). Es una titulación propia impartida por el Departament de Traducció, d'Interpretació i d'Estudis de l'Asia Oriental (DTIEAO) de la Universitat Autònoma de Barcelona (UAB) con una clara orientación profesionalizadora.

En este artículo nos proponemos describir los perfiles de prácticas que hemos identificado durante tres años académicos consecutivos (desde 2006 hasta 2009), al llevar a cabo las tareas de dirección y coordinación de dicho posgrado en el ámbito de la traducción jurídica en Barcelona, presentar los aspectos problemáticos y conflictivos de cada perfil, junto con nuestras propuestas de su solución.

Nuestro análisis de perfiles de prácticas se inspira en la clasificación y descripción de los siguientes ocho perfiles profesionales de traductores e intérpretes jurídicos, vigentes en el contexto catalán alrededor del año 2007 y recogidos por Torres Hostench ${ }^{1}$ : (1) traductor jurídico autónomo; (2) tra-

${ }^{1}$ O. Torres Hostench, "La inserció laboral dels llicenciats de traducció i interpretació en l'àmbit jurídic", Papers lextra, 3, 2007, pp. 63-71. 
ductor jurídico fijo en una empresa privada, no especializada en los servicios de traducción; (3) traductor judicial fijo de la Administración de Justicia; (4) traductor e intérprete jurado, habilitado por la Generalitat de Catalunya; (5) intérprete jurado nombrado por el Ministerio de Asuntos Exteriores; (6) intérprete judicial; (7) traductor de organismos internacionales (Comisión Europea, Parlamento Europeo, Tribunal de Justicia Europeo, organismos comunitarios descentralizados); y (8) intérprete de organismos internacionales (Parlamento Europeo, ONU).

\section{La formación de tercer ciclo en que se encuadra la oferta de prácticas no curriculares: el Postgrau de Traducció Jurídica de la Universitat Autònoma de Barcelona}

El PTJ ${ }^{2}$ es una titulación de tercer ciclo organizada por el DTIEAO de la UAB. Su oferta curricular incluye unos contenidos formativos de 40 créditos ECTS y un programa de prácticas optativas no curriculares.

\section{1. Áreas de formación}

El PTJ, con algunas variaciones en cuanto a sus contenidos formativos, se imparte de forma ininterrumpida desde el curso académico 2000/2001. El objetivo de esta titulación de tercer ciclo es proporcionar formación especializada en traducción jurídica a partir de una lengua extranjera (el inglés, el francés o el alemán) hacia el castellano o el catalán. El contenido del programa incluye conocimientos de los dos sistemas jurídicos correspondientes a los pares de lenguas seleccionados, el derecho comparado, el derecho internacional y el derecho europeo. Los contenidos teóricos representan un tercio de la formación total y están diseñados de forma que los alumnos adquieran los conocimientos jurídicos sin perder de vista las necesidades de la traducción ${ }^{3}$. Las asignaturas de práctica de la traducción jurídica ocupan los dos tercios restantes de la actividad formativa. El PTJ contempla únicamente asignaturas de traducción directa con objeto de poder reproducir en el aula prácticas

${ }^{2}$ Para más información: http://pagines.uab.cat/traducciojuridica/. Esta formación, desde el curso 2014/2015, se imparte dentro de un programa de Máster que aúna los conocimientos de traducción jurídica con la interpretación judicial y se puede consultar en esta página web: http:// pagines.uab.cat/tijuridica/es.

${ }^{3}$ C. Bestué, M. Orozco, "La necesidad de la naturalidad en la reformulación en la traducción jurídica en la 'era de la automatización' de las traducciones", JoSTrans. Journal of Specialised Translation, 15, 2011, pp. 180-199; C. Bestué, M. Orozco, "Online Training in legal Translation. Designing curricula for bilingual students”, Babel. International Journal of Translation, 62, 3, 2016, pp. 470-494. 
curriculares muy próximas a la realidad profesional ${ }^{4}$ y a los niveles de calidad que se esperan del traductor jurídico. En las asignaturas de traducción se trabaja con herramientas informáticas avanzadas, en salas de informática, y los contenidos tratados son encargos de traducción reales sobre textos de derecho internacional, europeo, civil, mercantil, patentes, etc. Se incluyen además contenidos sobre orientación profesional, seminarios específicos, conferencias y asistencia a sesiones de juicios entre otras actividades complementarias organizadas puntualmente.

Los alumnos que pueden acceder a esta titulación tienen tres perfiles diferenciados: licenciados en traducción e interpretación, licenciados en derecho y alumnos procedentes de otras licenciaturas, de preferencia filologías u otros estudios lingüísticos. Sin embargo, en algunas ediciones, el PTJ ha contado con perfiles más heterogéneos como licenciados en medicina, arquitectura o ingeniería, en cuyo caso han debido superar una prueba de acceso en la que deben demostrar un excelente dominio de la lengua de llegada y un perfecto dominio del inglés como lengua de partida.

La propuesta formativa ha ofrecido siempre las cinco combinaciones lingüísticas señaladas, es decir, inglés, francés y alemán, como lenguas de partida, y castellano y catalán como lenguas de llegada. No obstante, al tratarse de un título propio y por lo tanto sujeto a financiación mediante los ingresos procedentes de su propia matrícula, en la mayoría de las ediciones únicamente se ha impartido la combinación inglés-castellano. En dos ediciones fue posible desdoblar las sesiones de traducción hacia el catalán y hacia el castellano y también fue posible abrir un grupo de traducción francés-castellano.

\subsection{Caracterización de las prácticas}

En el PTJ, la oferta de prácticas es de dos tipos: (1) prácticas más breves, hasta un máximo de 90 horas, no remuneradas, y (2) prácticas más largas, hasta un máximo de 240, horas, remuneradas con un mínimo de $5 \mathrm{EUR} / \mathrm{hora}$ (según la recomendación de la oficina de Treball Campus UAB). El alumnado del PTJ solicita las prácticas mediante un formulario de solicitud y realiza, una vez terminadas, una evaluación de su experiencia de prácticas, también por escrito, utilizando un formulario preparado para este fin. Se trata de prácticas optativas y extracurriculares.

Las prácticas son un complemento a la formación que se ofrece en el PTJ, por ello, y porque de este modo en varios cursos se ha abierto la puerta hacia la contratación laboral de algunos alumnos, se ofrece la posibilidad de realizar las prácticas no estrictamente de traducción jurídica sino también de traduc-

${ }^{4}$ J. Engberg, "Comparative Law for Translation: The Key to Successful Mediation between Legal Systems", en: Legal Translation in Context. Professional Issues and Prospects, Oxford, Bern, Berlin, Bruxelles, Frankfurt am Main, New York, Wien, Peter Lang, 2013, pp. 9-27. 
ción muy vinculada a ella (sobre todo administrativa y financiera), en otra combinación lingüística y también en ámbitos del Derecho que el alumnado no ha tratado de forma directa en el PTJ aunque sí ha recibido un mínimo de base teórica. Por ejemplo, en la Fundación Ulls del Món los documentos que se elaboran (documentos para la solicitud de subvenciones) son del ámbito jurídico y forman parte del tipo de documentos frecuentes en entornos profesionales en los que se requiere una competencia multilingüe. Es importante que el alumnado comprenda que el PTJ es una base y que en el futuro deberían estar abiertos a todo tipo de documentos relacionados con este ámbito.

Las prácticas profesionales tienen una doble función: (1) la de formación en un lugar real de trabajo y (2) la de inserción laboral, proporcionando los primeros contactos con empresas e instituciones. De igual modo que existen perfiles profesionales en el ejercicio laboral de la traducción y la interpretación, existen también perfiles de prácticas, que reproducen toda la complejidad organizativa del mundo real, junto con los conflictos y tensiones internos y externos que ello conlleva.

\subsection{Coordinación de las prácticas: resolución de conflictos y diagnósti- co de la situación en el mercado}

La propia actividad de coordinación, definida por Terssac ${ }^{5}$ como construcción de un proceso complejo y coherente, realizable en unas condiciones dadas, a partir de tareas fragmentarias, pone de relieve la multiplicidad de subcomponentes de dicha actividad.

En primer lugar, un coordinador de prácticas debe asegurar el buen funcionamiento del sistema y de todas las demás funciones implicadas en el desarrollo de este tipo de formación: la representación institucional, la tutorización (docencia) y la gestión (entre otras funciones: archivo y certificación). Como señala $\mathrm{Zabalza}^{6}$, un buen funcionamiento de un sistema equivale a su calidad:

Buena calidad significa buena función..., o lo que es lo mismo, podemos decir que algo es de calidad cuando está cumpliendo con su función. Parece de perogrullo, pero ésa es la condición esencial de un buen PRACTICUM: que cumpla la función formativa que se le encomienda en un Plan de Estudios [...] ...y buena función significa buen trabajo. Los buenos resultados en procesos que implican la intervención de organizaciones y personas requieren de una acción combinada que lleve a la realización del plan previsto. Cada parte del sistema de los subsistemas implicados ha de estar cumpliendo adecuadamente su parte en la tarea conjunta. En caso contrario, todo el proceso se resentirá. [...] Hay muchas personas e instancias implicadas en su desarrollo. Sólo si cada una de ellas va cumpliendo su parte de acción colectiva se conseguirá que el proceso consiga su función.

${ }^{5}$ G. de Terssac, Autonomía en el trabajo, Madrid, Ministerio de Trabajo y Seguridad Social, 1995.

${ }^{6}$ M.A. Zabalza, "El estudio de las 'buenas prácticas' en la enseñanza universitaria”, $R E D U$. Revista de Docencia Universitaria, 10 (1), p. 22. 
Un elemento secundario de la coordinación, pero muy importante a la hora de conocer la situación en el mundo profesional de los futuros traductores jurídicos, consiste en recoger información acerca de las necesidades en servicios de traducción jurídica y de la formación que se requiere de los aspirantes (traductores jurídicos profesionales). El diagnóstico de la situación laboral en el sector de la traducción jurídica permite al coordinador de prácticas proponer medidas de adaptación de la gestión de las prácticas profesionalizadoras y, llegado el caso, en el programa de formación teórica en traducción jurídica del mismo posgrado ${ }^{7}$.

\section{Perfiles de prácticas}

En cuanto a las plazas de prácticas cubiertas durante tres cursos académicos $\left(2006 / 2007,2007 / 2008,2008 / 2009\right.$, Tabla $\left.1^{8}\right)$, observamos que en el curso 2006/2007 la mayoría de prácticas se realizó en despachos de abogados; en cambio, dos años después predominan las prácticas en despachos de traductores jurados, que interesaban sobre todo a los estudiantes que se preparaban para el examen de traductor jurado. Como una tendencia emergente podemos mencionar la constitución de un perfil de prácticas nuevo en el curso académico 2007/2008: los grupos de investigación en las universidades de Barcelona, distintos de la propia UAB. En la Tabla 1 se recogen estos datos cuantitativos correspondientes a las prácticas de PTJ realizadas por el alumnado en los años 2006-2009.

Tabla 1. Plazas de prácticas cubiertas, años 2006-2009, Postgrau de Traducció Juridica.

\begin{tabular}{|c|c|c|c|c|c|c|}
\hline \multirow{2}{*}{$\begin{array}{c}\text { Curso acadé- } \\
\text { mico }\end{array}$} & $\begin{array}{c}\text { Despachos } \\
\text { de trad. } \\
\text { jurados }\end{array}$ & $\begin{array}{c}\text { Despachos de } \\
\text { abogados }\end{array}$ & $\begin{array}{c}\text { Empresas de } \\
\text { traducción }\end{array}$ & $\begin{array}{c}\text { ONG, asocia- } \\
\text { ciones, insti- } \\
\text { tuciones }\end{array}$ & $\begin{array}{c}\text { Grupos de } \\
\text { investigación }\end{array}$ & TOTAL \\
\hline $2006 / 2007$ & 1 & 8 & 2 & 2 & 0 & 13 \\
\hline $2007 / 2008$ & 2 & 5 & 6 & 5 & 3 & 21 \\
\hline $2008 / 2009$ & 4 & 2 & 2 & 2 & 0 & 10 \\
\hline
\end{tabular}

El número total de estudiantes que realizaron las prácticas del PTJ oscila entre las 10 y las 21 personas. Esta diferencia se debe sobre todo a la variación en la matriculación general del posgrado en cada curso. El perfil de prácticas y la cantidad de plazas ocupadas por perfil y por cada curso académico

${ }^{7}$ A. Kuźnik, "Praktyki zawodowe jako źródło informacji o rynku pracy i usług z zakresu tłumaczeń prawniczych w Barcelonie", Rocznik Przekładoznawczy. Studia nad teoria, praktyka i dydaktyka przekładu, 9, 2014, pp. 303-315.

8 Véase también ibidem, p. 310. 
depende de varios factores, por ejemplo, por parte de los estudiantes: el interés por un perfil de prácticas en concreto, motivación personal, disponibilidad, condiciones materiales, experiencias laborales previas, etc.; y, por parte de las entidades: necesidades lingüísticas del momento, despachos y ordenadores disponibles, competencias y disponibilidad de tutores externos, recepción de estudiantes de prácticas de otras carreras y de otros centros académicos. Sin embargo, siempre alrededor del 80\% del alumnado está interesado en realizar esta actividad facultativa, que figura en su programa de posgrado.

A continuación presentamos la caracterización cualitativa de cada perfil y los conflictos, típicos de cada perfil, que hemos encontrado en los años 2006-2009. La información recogida y analizada proviene principalmente de los informes de prácticas redactados por los estudiantes al terminar la formación. Se ha tomado en cuenta también el contenido de los mensajes electrónicos intercambiados entre el coordinador, los tutores externos en las entidades receptoras y los estudiantes. Todos los casos han sido debidamente anonimizados y no reproducen datos de identificación reales.

\subsection{Despachos de traductores jurados}

En este perfil de prácticas, los estudiantes efectuaron traducciones oficiales, características de la actividad de un traductor jurado. Han trabajado con los siguientes documentos: textos jurídicos y académicos, documentos oficiales y certificados (certificados de antecedentes penales, partidas de matrimonio, partidas de nacimiento, títulos académicos y certificados escolares) y escrituras notariales (manifestaciones y poderes). Una de las estudiantes del curso 2008/2009 indica que ha trabajado principalmente con documentos procedentes de la India y de Pakistán, relacionados con los desplazamientos de los inmigrantes.

Los estudiantes han utilizado el inglés, el francés, el portugués, el español y el catalán. La combinación lingüística más común de las traducciones ha sido del inglés al español. En este perfil de prácticas, los estudiantes han aprovechado los conocimientos teóricos propios del derecho penal, administrativo, civil y mercantil.

En cuanto a las actividades realizadas, estas consistían en traducciones pero también en todas las demás actividades que acompañan la ejecución de los procesos de traducción. Uno de los estudiantes del curso académico 2008/2009 enumera las siguientes actividades:

He traducido los documentos que traían los clientes o que enviaban por correo electrónico o fax. Me documentaba para realizar las traducciones, realizaba búsquedas terminológicas y/o buscaba plantillas de documentos ya traducidos con anterioridad. Guardaba una base de datos con todos los documentos traducidos para reutilizarlos en su caso. Cuando terminaba se los entregaba al traductor jurado. También atendía a los clientes que venían a dejar el encargo o a 
retirar las traducciones, les resolvía las dudas y quejas. Por último, hacía consultas al traductor jurado cuando tenía dudas sobre terminología o formato del texto meta, así como otras de tipo profesional.

Los problemas más mencionados por los estudiantes en este perfil de prácticas han sido: (1) la necesidad de realizar traducciones a partir de textos manuscritos y por lo tanto muy poco legibles; y (2) la necesidad de efectuar traducciones indirectas, en las cuales los textos de partida eran a su vez traducciones y no documentos originales. Estos problemas han sido solucionados por los estudiantes con la ayuda y la supervisión de los traductores jurados para quienes trabajaban.

El coordinador de prácticas, sin embargo, tuvo que intervenir en varias situaciones relativas a la realización de las prácticas a distancia. Dado que en principio los despachos de traductores jurados cuentan con un reducido número de empleados y no disponen de mucho espacio, una parte de las prácticas ha sido realizada de manera no presencial, lo que conllevó sus dificultades de supervisión y de gestión, sobre todo en cuanto al cómputo de horas trabajadas.

\subsection{Despachos de abogados}

En los despachos de abogados, los estudiantes han realizado ante todo traducciones de textos jurídicos y de la correspondencia intercambiada entre los abogados y sus clientes extranjeros. Han utilizado en estas actividades el inglés, el alemán, el ruso, el francés, el español y el catalán. Se han apoyado en sus conocimientos teóricos de derecho procesal e internacional.

Entre las actividades realizadas especifican las siguientes: traducción y redacción de la correspondencia, de los escritos procesales y de la página web; revisión y corrección de traducciones realizadas previamente por otros estudiantes de prácticas, principalmente por los estudiantes de derecho (se trataba de corregir sus traducciones inversas al inglés); realización de llamadas esporádicas al extranjero para contactar con los clientes que hablaban uno de los idiomas antes mencionados. En sus informes finales de valoración de las prácticas, los estudiantes mencionaron tres traducciones concretas: (1) traducción de mensajes electrónicos del inglés al español y del español al ruso; (2) traducción de una sentencia del francés al español; y (3) traducción de un fragmento de la página web del despacho de abogados del español al inglés.

Dado que los despachos de abogados no son entidades especializadas en la prestación de los servicios de traducción, la problemática que encontramos giraba alrededor de la falta de recursos asignados a la realización y a la tutorización de las prácticas. Por esta razón, siempre sugeríamos a estos despachos la necesidad de asignar recursos suficientes para los estudiantes en prácticas (tutor, ordenador, programas electrónicos, acceso a glosarios, etc.). 


\subsection{Empresas de traducción}

La actividad más frecuente, llevada a cabo en las empresas de traducción en Barcelona, en este perfil de prácticas, ha sido la traducción de textos jurídicos. Dicho perfil ha sido solicitado principalmente por filólogos y licenciados en Derecho, es decir, por los estudiantes que hasta ese momento no han tenido mucho contacto con la traducción profesional.

Los textos traducidos han sido textos jurídicos y administrativos, principalmente certificados y documentos de registro civil (títulos académicos, certificados escolares, partidas de nacimiento y de matrimonio); contratos, cuentas anuales, citaciones y sentencias. Las combinaciones lingüísticas más comunes han sido: del inglés al español y del catalán al español. Puntualmente, los estudiantes han utilizado además el francés, el alemán, el italiano, el ruso y el portugués. Las áreas temáticas implicadas han sido el derecho de familia, penal y procesal.

Los estudiantes recogen las siguientes actividades: traducción; edición y revisión de textos; clasificación y preparación de textos para ser archivados, gestión de una base de datos de traducciones jurídicas previas; creación y actualización de glosarios. En las empresas de traducción las lenguas y los tipos de textos han sido más variados que en los despachos de bufetes de traductores jurados. Los estudiantes han trabajado menos con modelos de traducciones oficiales, y más con la edición de textos.

Los problemas de coordinación que han surgido en este perfil de prácticas han tenido una relación directa con la cuestión de la confidencialidad de los datos tratados. A veces las empresas requerían que los alumnos, en el momento de empezar las prácticas, firmasen los documentos de confidencialidad preparados por dichas empresas, sin haberlo consultado previamente con el coordinador del posgrado. En estos casos, recomendábamos recordar a la empresa de traducción que el convenio de prácticas incluye cláusulas especiales sobre la confidencialidad de los datos. Si aún así la empresa insistía en firmar su propio documento de confidencialidad, correspondía al alumno decidir si estaba de acuerdo en firmarlo. Si lo firmaba, se adjuntaba una copia a su expediente de prácticas. Si el alumno no estaba de acuerdo con firmarlo, las prácticas debían ser anuladas por causa de la empresa, ya que esta no había informado antes al coordinador de prácticas sobre este requisito previo.

\subsection{Organizaciones no gubernamentales, asociaciones e instituciones}

En las organizaciones no gubernamentales (ONG), asociaciones e instituciones culturales los estudiantes han trabajado con textos jurídicos, periodísticos, informativos y didácticos. Los estudiantes trabajaron con el catalán, el español, el inglés y el francés en las siguientes combinaciones lingüísticas: 
del catalán al español (y al revés), del inglés al español y al catalán, del español al inglés y del español al francés, del francés al catalán (y al revés). Los textos traducidos se basaban en el derecho de trabajo, derecho internacional, derechos humanos y cuestiones relacionadas con la migración.

Concretamente, las prácticas en este perfil consistían en las siguientes actividades: traducción y edición de la página web; actualización y revisión del documento "Plan de acción para 2008" en el cual se exponían las actividades de una ONG previstas para el año 2008; preparación de una presentación multimedia con informe de actividades llevadas al cabo por dicha ONG a lo largo del año 2008; creación de un glosario de términos especializados; y participación en la redacción de un material didáctico (indicaciones para los voluntarios sobre cómo traducir). Una de las actividades predominantes ha sido la revisión y corrección de textos originales (en catalán) y de las traducciones realizadas con anterioridad (del español al catalán, del catalán al francés, etc.). La compilación de traducciones fragmentarias previas, su unificación terminológica, estilística y gráfica, y su composición en una sola obra (un manual) ha sido una actividad muy común, sobre todo en estas entidades que no suelen emplear para dichas tareas redactores y traductores profesionales sino que aprovechan la participación esporádica de estudiantes de prácticas.

Para el coordinador de prácticas, este perfil de prácticas resultó conflictivo debido a la inadecuación del perfil a las expectativas de los estudiantes, sobre todo en cuanto a la abundancia de la actividad de revisión frente a la de traducción. Sin embargo, por tratarse de un ámbito muy operacional, en el que el traductor tenía la consideración de verdadero experto en las tareas de traducción, las cuales eran compatibilizadas con tareas de revisión, de redacción multilingüe, tales como informes y comunicados, además de la elaboración de glosarios de la terminología más utilizada en la ONG, resultaron ser una actividad formativa muy apreciada especialmente por las personas que contaban con experiencia previa en otros contextos.

\subsection{Grupos de investigación en centros académicos}

En el curso académico 2007/2008, a raíz de una nueva necesidad detectada, surgió un nuevo perfil de prácticas, es decir, las prácticas realizadas en los grupos de investigación vinculadas con las facultades de derecho en otros centros académicos que la UAB.

Las lenguas de trabajo utilizadas han sido similares a los perfiles anteriores: español, catalán, inglés, francés, alemán e italiano. Esta vez los estudiantes han trabajado con textos de carácter científico-académico, por ejemplo, presentaciones en congresos, artículos, informes de los grupos de investigación, documentos para las convocatorias de financiación, etc. Ha sido de gran utilidad el conocimiento del derecho penal e internacional debido a los intere- 
ses académicos muy específicos de los grupos de investigación que acogieron los estudiantes del PTJ.

Las actividades consistían en traducciones, búsqueda de información, preparación de materiales informativos y didácticos. En el fondo, en este perfil de prácticas, los estudiantes combinaban tres áreas: la traducción, el derecho y la investigación.

Tanto los estudiantes como los propios investigadores en los grupos de investigación implicados mencionaron en este perfil una sola dificultad básica: su escasa capacidad de inserción laboral de nuestros estudiantes de prácticas, ya que la contratación en los grupos de investigación sigue su propio funcionamiento, trazado por la vía de contratación en establecimientos públicos de enseñanza superior.

\section{Conclusiones}

En primer lugar, pensamos que cabe completar la propuesta de clasificación de perfiles profesionales en traducción e interpretación jurídica, propuesta por Torres Hostench ${ }^{9}$ e incluir en dicha tipologías tres de los perfiles identificados por nosotros: traductores (e intérpretes) en empresas de traducción, en organizaciones no gubernamentales y en grupos de investigación académica.

En segundo lugar, hemos comprobado el carácter muy heterogéneo de las actividades realizadas en cada perfil de prácticas en traducción jurídica. Dicha naturaleza híbrida ha sido ya mencionada por los especialistas en la profesión de traductor jurídico y jurado en España ${ }^{10}$, y también por nosotros en estudios previos, relacionados con los puestos de trabajo de traductores internos no especializados ${ }^{11}$. Dicha heterogeneidad se refiere tanto a la variedad de tareas realizadas como a los tipos de textos manejados y las lenguas implicadas.

En tercer lugar, los conflictos que surgieron durante la coordinación de las prácticas ponen de manifiesto la naturaleza muy compleja de estas actividades formativas desde varios puntos de vista. Tras nuestro análisis podemos concluir que los conflictos pueden emanar de las siguientes fuentes:

${ }^{9}$ O. Torres Hostench, op. cit.

${ }^{10}$ Cfr. R. Mayoral, "El polifacetismo del traductor (jurídico y jurado)", en: C. García, I. García (eds.), Experiencias de traducción. Reflexiones desde la práctica traductora, Castellón de la Plana, Publicacions de la Universitat Jaume I, 2004, pp. 165-180.

${ }^{11}$ A. Kuźnik, "Puestos de trabajo híbridos. Cuatro indicadores del carácter heterogéneo de los puestos de trabajo internos en traducción", Sendebar, 22, 2011, pp. 283-307; El contenido de los puestos de trabajo de los traductores. El caso de los traductores internos en las empresas de traducción de Barcelona, Saarbrücken: AV Akademikerverlag GmbH \& Co. KG/ Editorial Académica Española, 2012, 590 p.; "Work content of in-house translators in small and medium-sized industrial enterprises. Observing real work situations", JoSTrans. The Journal of Specialised Translation, 25, 2016 (January), pp. 213-231; A. Kuźnik, J.M. Verd, "Investigating Real Work Situations in Translation Agencies. Work Content and its Components", Hermes, 44, 2010, pp. 25-43. 
1. Desconocimiento de la entidad y del perfil de prácticas por parte de los estudiantes.

2. Enorme variabilidad de los actores implicados en la ejecución de las prácticas.

3. Fallos en los eslabones del proceso: cada componente de esta compleja cadena de gestión de las prácticas puede fallar en cada momento y tenemos que saber responder a ello.

4. Intereses implícitos: tanto por parte de los estudiantes como por parte de las entidades que ofrecen prácticas, existen siempre intereses y motivaciones implícitos, tácitos, que solo se manifiestan cuando un elemento de la gestión empieza a fallar.

5. Diferencia de factores por los cuales se rigen los estudiantes y las entidades de prácticas: para los estudiantes estos factores son frecuentemente emocionales (una apuesta por un futuro trabajo) y para las entidades de prácticas suelen ser factores empresariales (costes, rentabilidad e imagen).

Además, Zabalza ${ }^{12}$ nos recuerda que al margen de cuál sea la estructura y los propósitos formativos del "practicum", éste tiene siempre una dimensión personal que desborda ampliamente los objetivos planificables. Cada sujeto va a vivir el "practicum" de una manera particular, manera que en parte dependerá de la propia organización del periodo de prácticas y, en parte, del talante con que cada estudiante afronte y se involucre en la experiencia. Dicha dimensión personal de las prácticas la convierte en una experiencia única y singular, difícilmente predecible por el coordinador de prácticas y por sus tutores.

Basándonos en el análisis llevado a cabo, podemos proponer las siguientes estrategias para enfocar y solucionar los conflictos emergentes:

1. Informar a los estudiantes sobre el perfil de prácticas que seguirán: dar a conocer las entidades de prácticas antes, en una reunión previa con los alumnos, distribuir su documentación y presentar sus formas de trabajo.

2. Establecer metas reales y ser conscientes de que siempre habrá conflictos nuevos; el gran reto es reducir su número y suavizar su impacto, y no eliminarlos del todo, que sería una vana ilusión. Algunos conflictos y tensiones no se solucionan nunca y hay que asumir su presencia a largo plazo; otros conflictos se solucionarán más tarde y por ello vale la pena registrarlos y tenerlos presentes. Como bien afirma Zabalza ${ }^{13}$, "Ninguna práctica es buena en todos sus componentes [como] [n]ingún profesor/a es un compendio de buenas prácticas."

3. Más vale prevenir que curar: anticipar los conflictos siempre cuando se pueda, ser un actor activo y consciente de la colaboración es decir dedicar tiempo a las primeras fases de planificación e implementación, leer los

\footnotetext{
12 M.A. Zabalza, op. cit.

13 Ibidem, p. 19.
} 
informes y los mensajes electrónicos detenidamente, establecer políticas de comunicación y gestión a largo plazo.

4. Fomentar la confianza y el trabajo de equipo; utilizar canales y herramientas de comunicación adecuadas y respetuosas.

5. Acortar el tiempo de reacción; los conflictos deben solucionarse con rapidez, antes de que crezcan y tomen una dimensión desmesurada e incontrolable.

6. Tener la conciencia del sistema: la gestión y el funcionamiento de las prácticas forman un sistema complejo en el cual no hay eventos aislados sino que todos los eslabones están interconectados; mediante la gestión de los conflictos, hay que ir descubriendo las interdependencias entre ellos.

7. Aplicar el trabajo emocional: no perder los nervios al tratar las situaciones conflictivas y pensar que siempre hay una solución para todo.

8. Enfocar los conflictos como motivación para mejorar el sistema, dado que cada conflicto permite detectar debilidades, avanzar y mejorar en algún aspecto importante de nuestro trabajo.

\section{Referencias bibliográficas}

BESTUÉ C., OROZCO M.

2011 "La necesidad de la naturalidad en la reformulación de la traducción jurídica en la 'era de la automatización' de las traducciones”, JoSTrans. The Journal of Specialised Translation, 15, pp. 180-199.

2016 "Online Training in legal Translation. Designing curricula for bilingual students", Babel. International Journal of Translation, 62, 3, pp. 470-494. DOI: 10.1075/babel.62.3.06bes. ENGBERG J.

2013 "Comparative Law for Translation: The Key to Successful Mediation between Legal Systems", en: Borja Albi A., Prieto Ramos F. (eds.), Legal Translation in Context. Professional Issues and Prospects, Oxford, Bern, Berlin, Bruxelles, Frankfurt am Main, New York, Wien, Peter Lang, vol. 4, pp. 9-27.

KUŹNIK A.

2011 "Puestos de trabajo híbridos. Cuatro indicadores del carácter heterogéneo de los puestos de trabajo internos en traducción", Sendebar, 22, pp. 283-307.

2012 El contenido de los puestos de trabajo de los traductores. El caso de los traductores internos en las empresas de traducción de Barcelona, Saarbrücken, AV Akademikerverlag $\mathrm{GmbH} \& \mathrm{Co} . \mathrm{KG}$ - Editorial Académica Española.

2014 "Praktyki zawodowe jako źródło informacji o rynku pracy i usług z zakresu thumaczeń prawniczych w Barcelonie", Rocznik Przekładoznawczy. Studia nad teoria, praktyka i dydaktyka przektadu, 9, pp. 303-315.

2016 "Work content of in-house translators in small and medium-sized industrial enterprises. Observing real work situations", JoSTrans. The Journal of Specialised Translation, 25, pp. 213-231.

KUŹNIK A., VERD J.M.

2010 "Investigating Real Work Situations in Translation Agencies. Work Content and its Components", Hermes, 44, pp. 25-43. 
MAYORAL ASENSIO R.

2004 "El polifacetismo del traductor (jurídico y jurado)", en: García C., García I. (eds.), Experiencias de traducción. Reflexiones desde la práctica traductora, Castellón de la Plana, Publicacions de la Universitat Jaume I, pp. 165-180.

TERSSAC G. de

1995 Autonomía en el trabajo, Madrid, Ministerio de Trabajo y Seguridad Social.

TORRES HOSTENCH O.

2007 "La inserció laboral dels llicenciats de traducció i interpretació en l'àmbit jurídic", Papers lextra, 3, pp. 63-71.

ZABALZA BAREZA M.Á.

2012 "El estudio de las 'buenas prácticas' en la enseñanza universitaria”, REDU. Revista de Docencia Universitaria, 10 (1), pp. 17-42.

\section{Work placements profiles and emerging conflicts resolution: Case study of work placements in legal translation}

Keywords: legal translation — postgraduate diploma in legal translation - work placements — placement profiles - conflict resolution - coordination.

\section{Abstract}

This article presents a qualitative analysis of the optional work placements - envisaged here as a learning activity — that form part of the Postgraduate Diploma in Legal Translation offered by the Department of Translation, Interpreting and East Asian Studies at the Universitat Autònoma de Barcelona. The authors divide the placements into five profiles, according to the type of organisation hosting the students on the programme: sworn translation providers, law firms, translation agencies, non-governmental organisations such as associations and institutions and, finally, university research groups. The analysis is followed by an examination of the conflicts arising in each profile and proposals for dealing with these. In their conclusions, the authors state that the heterogeneous nature of the work placements in specialised legal translation should be seen as an argument supporting the same heterogeneous nature of work content in non-specialised, general translation. 